\title{
Oxidation Potentials Correlate with Conductivities of Aromatic Molecular Wires
}

\author{
Jordan R. Quinn, Frank W. Foss Jr., Latha Venkataraman,* and Ronald Breslow* \\ Department of Chemistry, Department of Physics, and Center for Electron Transport in \\ Molecular Nanostructures, Columbia University, New York, New York 10027
}

\section{Supplementary Information}

\section{Synthetic Procedures \\ 2. Electrochemistry Methods \\ 3. CV Data and Voltammograms}

\section{Synthetic Procedures:}

Reagents and starting materials obtained from commercial sources were typically $\geq 97 \%$ purity and were used without further purification unless stated otherwise. 2,6-Diaminonaphthalene, ${ }^{1}$ 2,6-diaminoanthracene ${ }^{2}$ and 9,10-diaminoanthracene ${ }^{3}$ were synthesized according to published methods and were sublimed under vacuum $\left(<200^{\circ} \mathrm{C}\right)$ prior to use.

4,4'-(1,4-naphthalenediyl)bisbenzamine (8). To a solution of $188 \mathrm{mg}(0.657 \mathrm{mmol})$ of 1,4dibromonaphthalene in $10 \mathrm{~mL}$ of toluene was added $120 \mathrm{mg}(0.104 \mathrm{mmol})$ of tetrakis(triphenylphosphine) palladium and the mixture was stirred at room temperature for 30 min. To the solution was added $1.3 \mathrm{~mL}(2 \mathrm{M}, 2.6 \mathrm{mmol})$ of aqueous sodium carbonate followed by $288 \mathrm{mg}(1.31 \mathrm{mmol})$ of 4-(4,4,5,5-tetramethyl-1,3,2-dioxaborolan-2-yl)-aniline (Aldrich) in $10 \mathrm{~mL}$ of ethanol. The resulting mixture was heated at reflux for $5.5 \mathrm{~h}$. The mixture was cooled to room temperature and partitioned between $25 \mathrm{~mL}$ of water and $25 \mathrm{~mL}$ of ethyl acetate. The organic layer was removed and the aqueous layer was extracted with $25 \mathrm{~mL}$ of ethyl acetate. The combined organic layers were dried over sodium sulfate, filtered, concentrated in vacuo and purified by flash chromatography on silica gel using 3:1 hexane:ethyl acetate to 1:1 hexane:ethyl acetate. The oily product fraction was dissolved in $2 \mathrm{~mL}$ of ethyl acetate and was treated with ca. $10 \mathrm{~mL}$ of hexanes until the solution became slightly cloudy. The product precipitated as a pale yellow solid ( $91 \mathrm{mg}, 45 \%$ yield). A portion was sublimed at $200-220^{\circ} \mathrm{C}$ under vacuum for conductance and electrochemical measurements. ${ }^{1} \mathrm{H} \mathrm{NMR}\left(\mathrm{CDCl}_{3}, 400 \mathrm{MHz}\right) \delta 8.03-8.01(\mathrm{~m}$, 2H), 7.42-7.32 (m, 8H), 6.84-6.82 (m, 4H), 3.77 (br s, 4H); APCI-MS (MeOH/Me $\left.{ }_{2} \mathrm{CO}\right): 311$ $\left(\mathrm{M}+\mathrm{H}^{+}\right)$.

\footnotetext{
${ }^{1}$ Chatt, J.; Wynne, W. P. J. Chem. Soc. 1943, 33-36.

${ }^{2}$ Rabjohns, M. A.; Hodge, P.; Lovell, P. A. Polymer 1997, 38, 3395-3407.

${ }^{3}$ Schiedt, v. B. J. Prakt. Chem. 1941, 157, 203-224. Campbell, T. W.; Mccoy, V. E.; Kauer, J. C.; Foldi, V. S. J.

Org. Chem. 1961, 26, 1422-1426.
} 


\section{Electrochemistry Methods:}

Electrochemistry was performed on a BAS CV-50W voltammetric analyzer with a three electrode cell. Each diamine was dissolved in acetonitrile containing $0.1 \mathrm{M}$ tetrabutylammonium perchlorate (TBAP) as supporting electrolyte to give $1.0 \mathrm{mM}$ final concentration in diamine. Sample solutions were sparged with argon for 5 min. prior to use. A platinum disc working electrode (BAS, $\mathrm{d}=1.6 \mathrm{~mm}$ ) and platinum wire counter electrode were used. The reference electrode contained a silver wire with $10 \mathrm{mM}$ silver nitrate in TBAP electrolyte solution. Cyclic voltammetry experiments were carried out at ambient temperature and were recorded at $100 \mathrm{mV}$ $\mathrm{s}^{-1}$. Potentials were swept from $-0.5 \mathrm{~V}$ to $+2.0 \mathrm{~V}$ or from $-1.5 \mathrm{~V}$ to $+1.5 \mathrm{~V}$. Ferrocene was used as an external calibrator and gave $E_{1 / 2}=90 \mathrm{mV}$ (vs $\mathrm{Ag} / \mathrm{AgNO}$ ) for each experiment.

\section{Conductance Data, CV Data and Voltammograms ${ }^{a}$ :}

\begin{tabular}{lcccc}
\hline \multicolumn{1}{c}{ compound } & number & $\begin{array}{c}\text { conductance } \\
\left(G_{0} \times 10^{-3}\right)\end{array}$ & $\begin{array}{c}E_{1 / 2}(\mathrm{~V}) \\
\text { vs Fc/Fc }\end{array}$ & $\begin{array}{c}\text { distance } \\
\mathrm{N}-\mathrm{N}(\AA)\end{array}$ \\
\hline diaminophenylnaphthalene & $\mathbf{8}$ & 0.035 & 0.370 & 14.2 \\
terphenyldiamine & $\mathbf{7}$ & 0.18 & 0.262 & 14.2 \\
tetrachlorobenzidine & $\mathbf{S 1 1}$ & 0.4 & 0.870 & 9.9 \\
octafluorobenzidine & $\mathbf{1 0}$ & 0.5 & 1.088 & 10.0 \\
dichlorobenzidine & $\mathbf{S 1 2}$ & 0.9 & 0.371 & 9.9 \\
benzidine & $\mathbf{6}$ & 1.1 & 0.133 & 9.9 \\
tolidine & $\mathbf{S 1 3}$ & 1.3 & 0.084 & 9.9 \\
tetramethylbenzidine & $\mathbf{S 1 4}$ & 1.4 & 0.042 & 9.9 \\
2,7-diaminofluorene & $\mathbf{S 1 5}$ & 1.5 & -0.037 & 9.6 \\
2,6-diaminoanthracene & $\mathbf{5}$ & 1.6 & -0.020 & 10.1 \\
2,6-diaminonaphthalene & $\mathbf{4}$ & 2.6 & -0.024 & 7.8 \\
tetrafluorphenylenediamine & $\mathbf{9}$ & 5.5 & 0.400 & 5.6 \\
chlorophenylenediamine & $\mathbf{S 1 6}$ & 6.0 & 0.052 & 5.6 \\
cyanophenylenediamine & $\mathbf{S 1 7}$ & 6.0 & 0.170 & 5.6 \\
dichlorophenylenediamine & $\mathbf{S 1 8}$ & 6.1 & 0.217 & 5.6 \\
trifluoromethylphenylenediamine & $\mathbf{S 1 9}$ & 6.1 & 0.120 & 5.6 \\
1,4-diaminobenzene & $\mathbf{3}$ & 6.4 & -0.122 & 5.6 \\
dimethylphenylenediamine & $\mathbf{S 2 0}$ & 6.7 & -0.189 & 5.6 \\
chloromethylphenylenediamine & $\mathbf{S 2 1}$ & 7.1 & 0.019 & 5.6 \\
tetramethylphenylenediamine & $\mathbf{S 2 2}$ & 8.2 & -0.259 & 5.6 \\
1,4-diaminonaphthalene & $\mathbf{2}$ & 9.0 & -0.220 & 5.6 \\
9,10-diaminoanthracene & $\mathbf{1}$ & 17 & -0.412 & 5.6 \\
\hline & & & & \\
\hline
\end{tabular}

${ }^{a}$ Conductivities $\left(G_{0} \times 10^{-3}\right)$ are generally within $5 \%$ of the indicated value. Half-wave potentials $\left(E_{1 / 2}\right)$ are first oxidation potentials $( \pm 10 \mathrm{mV})$. Italicized $E_{1 / 2}$ values were estimated by subtracting $59 / 2 \mathrm{mV}$ from first anodic peak potentials when irreversibility was observed (where $59 \mathrm{mV}$ is the expected difference between the first anodic peak potential and the second cathodic peak potential for a one-electron redox process). Nitrogen-to-nitrogen (N-N) distances were approximated from MMFF-minimized geometries. ${ }^{4}$

\footnotetext{
${ }^{4}$ Spartan '04, Wavefunction, Inc. Irvine, CA.
} 
In the voltammograms below, the $\mathrm{y}$-axis is current $(\mathrm{A})$ and $\mathrm{x}$-axis is potential $(E, \mathrm{mV}$ vs. $\mathrm{Ag} / \mathrm{Ag}^{+}$):
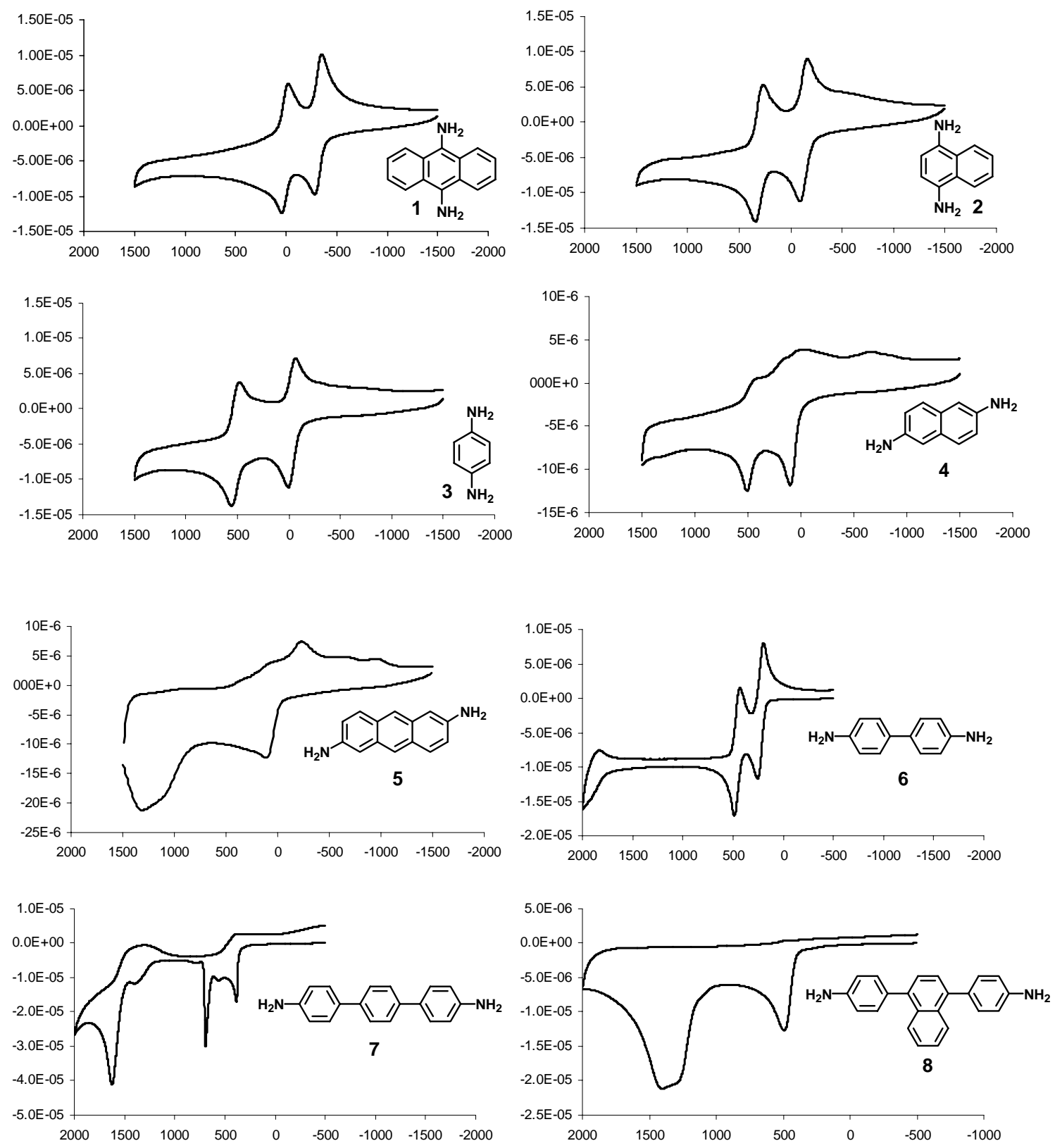

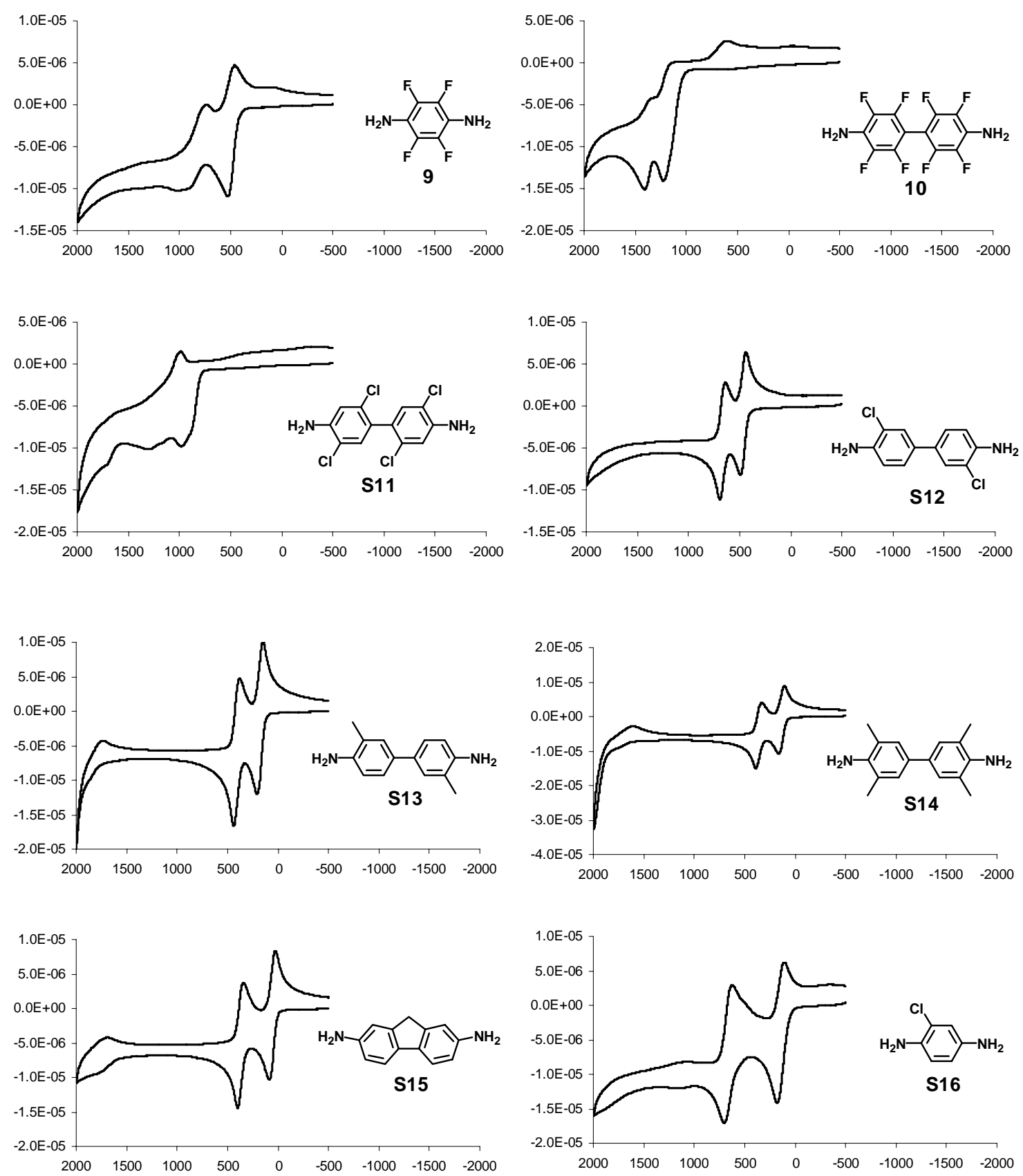

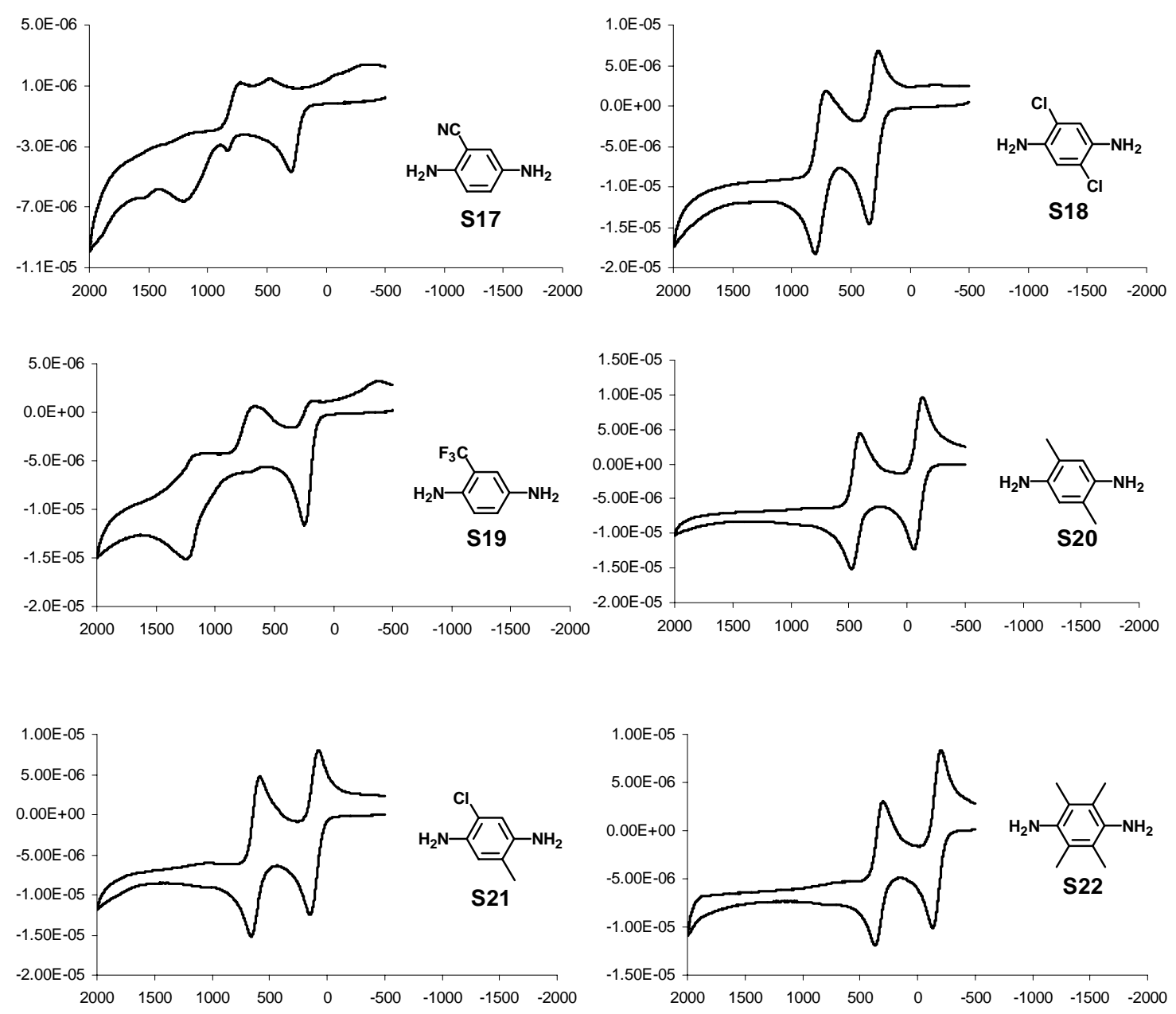